J. Nonlinear Var. Anal. 5 (2021), No. 1, pp. 141-153

Available online at http://jnva.biemdas.com

https://doi.org/10.23952/jnva.5.2021.1.09

\title{
VOLTERRA INTEGRAL OPERATORS AND CARLESON EMBEDDING ON CAMPANATO SPACES
}

\author{
XIAOSONG LIU ${ }^{1}$, SONGXIAO LI ${ }^{2, *}$, RUISHEN QIAN ${ }^{3}$ \\ ${ }^{1}$ Department of Mathematics, Shantou University, Shantou 515063, China \\ ${ }^{2}$ Institute of Fundamental and Frontier Sciences, \\ University of Electronic Science and Technology of China, Chengdu 610054, China \\ ${ }^{3}$ School of Mathematics and Statistics, Lingnan Normal University, Zhanjiang 524048, China
}

\begin{abstract}
Let $1 \leq p<q<\infty$. The boundedness and compactness of the embedding from Campanato spaces $\mathscr{L}_{p, \lambda}$ into tent spaces $\mathscr{T}_{s}^{q}(\mu)$ are investigated in this paper. Meanwhile, the boundedness and the essential norm of the Volterra operators $T_{g}$ and $I_{g}$ from $\mathscr{L}_{p, \lambda}$ to $F\left(q, q-2+\frac{q(1-\lambda)}{p}, s\right)$ are also studied.

Keywords. Campanato space; Volterra integral operator; Embedding; Carleson measure.
\end{abstract}

\section{INTRODUCTION}

Let $\mathbb{D}$ denote the open unit disk in the complex plane $\mathbb{C}$ and $\mathscr{H}(\mathbb{D})$ denote the space of all analytic functions in $\mathbb{D}$. As usual, $\mathscr{H}^{\infty}$ denotes the space of bounded analytic functions. Let $p \geq 1$ and $0<\lambda<\infty$. We say that an $f \in H^{p}$ belongs to the analytic Campanato spaces $\mathscr{L}_{p, \lambda}$ if

$$
\|f\|_{\mathscr{L}_{p, \lambda}}^{p}=\sup _{I \subset \partial \mathbb{D}} \frac{1}{|I|^{\lambda}} \int_{I}\left|f(\zeta)-f_{I}\right|^{p} \frac{|d \zeta|}{2 \pi}<\infty
$$

where

$$
f_{I}=\frac{1}{|I|} \int_{I} f(\zeta) \frac{|d \zeta|}{2 \pi}, I \subseteq \partial \mathbb{D},
$$

and the Hardy space $H^{p}$ (see [1]) consists of all $f \in H(\mathbb{D})$ such that

$$
\|f\|_{H^{p}}^{p}=\sup _{0<r<1} \frac{1}{2 \pi} \int_{0}^{2 \pi}\left|f\left(r e^{i \theta}\right)\right|^{p} d \theta<\infty .
$$

If $p=2$, then space $\mathscr{L}_{2, \lambda}$ is called the Morrey space, which was first studied by Wu and Xie [2] in the case of $\mathbb{D}$. We refer to [3,4] for more study on the Morrey space $\mathscr{L}_{2, \lambda}$. If $p=2$ and $\lambda=1$, then $\mathscr{L}_{2, \lambda}$ is just the BMOA space. For more information on $B M O A$ space, we refer to $[5]$.

${ }^{*}$ Corresponding author.

E-mail addresses: gdxsliu@163.com (X. Liu), jyulsx@163.com (S. Li), qianruishen@sina.cn (R. Qian).

Received November 2, 2020; Accepted January 7, 2021.

(C)2021 Journal of Nonlinear and Variational Analysis 
Suppose that $0<p<\infty,-2<q<\infty$ and $0<s<\infty$. The space $F(p, q, s)$ is defined by those $f \in \mathscr{H}(\mathbb{D})$ with

$$
\|f\|_{F(p, q, s)}^{p}=|f(0)|^{p}+\sup _{a \in \mathbb{D}} \int_{\mathbb{D}}\left|f^{\prime}(z)\right|^{p}\left(1-|z|^{2}\right)^{q}\left(1-\left|\varphi_{a}(z)\right|^{2}\right)^{s} d A(z)<\infty,
$$

where $\varphi_{a}(z)=\frac{a-z}{1-\bar{a} z}$. This space was first introduced by Zhao [6]. If $p=2$ and $q=0$, it gives $Q_{s}$ spaces (see [7]). From [6], we see that $F(p, p-2, s)$ is equivalent to a Bloch space for all $s>1$, where the Bloch space $\mathscr{B}$ is the class of all $f \in \mathscr{H}(\mathbb{D})$ such that

$$
\|f\|_{\mathscr{B}}=|f(0)|+\sup _{z \in \mathbb{D}}\left(1-|z|^{2}\right)\left|f^{\prime}(z)\right|<\infty .
$$

The little Bloch space $\mathscr{B}_{0}$ consists of all $f \in \mathscr{H}(\mathbb{D})$ for which

$$
\lim _{|z| \rightarrow 1^{-}}\left(1-|z|^{2}\right)\left|f^{\prime}(z)\right|=0 .
$$

Let $\partial \mathbb{D}$ denote the boundary of $\mathbb{D}$. Let $I$ be an arc of $\partial \mathbb{D}$ and $|I|$ be the normalized Lebesgue arc length of $I$. The Carleson square based on $I$, denoted by $S(I)$, is defined by

$$
S(I):=\left\{z=r e^{i \theta} \in \mathbb{D}: 1-|I| \leq r<1, e^{i \theta} \in I\right\} .
$$

Let $0<\alpha<\infty$, and let $\mu$ be a positive Borel measure on $\mathbb{D}$. Recall that $\mu$ is called an $\alpha$-Carleson measure if

$$
\sup _{I \subset \partial \mathbb{D}} \frac{\mu(S(I))}{|I|^{\alpha}}<\infty .
$$

If $\mu$ is an $\alpha$-Carleson measure, then we set

$$
\|\mu\|_{\alpha}=\sup _{I \subset \partial \mathbb{D}} \frac{\mu(S(I))}{|I|^{\alpha}}
$$

If $\lim _{|I| \rightarrow 0} \frac{\mu(S(I))}{|I|^{\alpha}}=0$, then $\mu$ is called a vanishing $\alpha$-Carleson measure.

Let $\mu$ be a positive Borel measure on $\mathbb{D}$. For $0<q<\infty$ and $0<s<\infty$, the tent space $\mathscr{T}_{s}^{q}(\mu)$ consists of all $\mu$-measurable functions $f$ such that

$$
\|f\|_{\mathscr{T}^{q}(\mu)}^{q}=\sup _{I \subset \partial \mathbb{D}} \frac{1}{|I|^{s}} \int_{S(I)}|f(z)|^{q} d \mu(z)<\infty .
$$

For more information about some function spaces embedding into tent space $\mathscr{T}_{s}^{q}(\mu)$, we refer to $[4,8,9,10]$ and the references therein.

In this paper, we study the embedding of $\mathscr{L}_{p, \lambda}$ into $\mathscr{T}_{s}^{q}(\mu)$ when $1 \leq p<q<\infty$. That is, we prove that the identity mapping $i: \mathscr{L}_{p, \lambda} \rightarrow \mathscr{T}_{s}^{q}(\mu)$ is bounded (resp.compactly) if and only if

$$
\sup _{I \subset \partial \mathbb{D}} \frac{\mu(S(I))}{|I|^{s+\frac{q(1-\lambda)}{p}}}<\infty\left(\operatorname{resp} . \lim _{|I| \rightarrow 0} \frac{\mu(S(I))}{|I|^{s+\frac{q(1-\lambda)}{p}}}=0\right),
$$

when $0<\lambda<1$ and $\frac{q \lambda}{p}<s<\infty$. Furthermore, the boundedness, the compactness and the essential norm of operators $T_{g}$ and $I_{g}$ from $\mathscr{L}_{p, \lambda}$ to $F\left(q, q-2+\frac{q(1-\lambda)}{p}, s\right)$ are also investigated.

Throughout this paper, let $f$ and $g$ be two positive functions, and write $f \lesssim g$ if $f \leq C g$ holds, where $C$ is a positive constant independent of $f$ and $g$. If $f \lesssim g$ and $g \lesssim f$, then we say $f \asymp g$. 


\section{EMbedding $\mathscr{L}_{p, \lambda}$ INTO TENT SPACES $\mathscr{T}_{s}^{q}(\mu)$}

In this section, we investigate the boundedness and compactness of the identity mapping $i: \mathscr{L}_{p, \lambda} \rightarrow \mathscr{T}_{s}^{q}(\mu)$. First, let us recall the following result, which can be found in [8].

Lemma 2.1. Let $\mu$ be a positive Borel measure on $\mathbb{D}$. If $0<\alpha, t<\infty$, then $\mu$ is an $\alpha$-Carleson measure if and only if

$$
\sup _{a \in \mathbb{D}} \int_{\mathbb{D}} \frac{\left(1-|a|^{2}\right)^{t}}{|1-\bar{a} z|^{\alpha+t}} d \mu(z)<\infty
$$

Moreover,

$$
\sup _{I \subset \partial \mathbb{D}} \frac{\mu(S(I))}{|I|^{\alpha}} \asymp \sup _{a \in \mathbb{D}} \int_{\mathbb{D}} \frac{\left(1-|a|^{2}\right)^{t}}{|1-\bar{a} z|^{\alpha+t}} d \mu(z) .
$$

Lemma 2.2. [9] Let $1 \leq p<\infty$ and $0<\lambda<1$. If $f \in \mathscr{L}_{p, \lambda}$, then

$$
|f(z)| \lesssim \frac{\|f\|_{\mathscr{L}_{p, \lambda}}}{\left(1-|z|^{2}\right)^{\frac{1-\lambda}{p}}}, z \in \mathbb{D} .
$$

Lemma 2.3. [11] For $0<r<1$, let $\chi_{\{z:|z|<r\}}$ be the characteristic function of the set $\{z:|z|<r\}$. If $\mu$ is a $\alpha$-Carleson measure on $\mathbb{D}$, then $\mu$ is a vanishing $\alpha$-Carleson measure if and only if $\left\|\mu-\mu_{r}\right\|_{\alpha} \rightarrow 0$ as $r \rightarrow 1^{-}$, where $d \mu_{r}=\chi_{\{z:|z|<r\}} d \mu$.

Now, we are in a position to prove the main result in this section.

Theorem 2.1. Let $\mu$ be a positive Borel measure on $\mathbb{D}$. Let $1 \leq p<q<\infty, 0<\lambda<1$ and $\frac{q \lambda}{p}<s<\infty$. Then the identity mapping $i: \mathscr{L}_{p, \lambda} \rightarrow \mathscr{T}_{s}^{q}(\mu)$ is bounded if and only if

$$
\sup _{I \subset \partial \mathbb{D}} \frac{\mu(S(I))}{|I|^{s+\frac{q(1-\lambda)}{p}}}<\infty .
$$

Proof. Assume that $i: \mathscr{L}_{p, \lambda} \rightarrow \mathscr{T}_{s}^{q}(\mu)$ is bounded. For $a \in \mathbb{D}$, set

$$
f_{a}(z)=\frac{\left(1-|a|^{2}\right)^{1+\frac{\lambda-1}{p}}}{(1-\bar{a} z)}, z \in \mathbb{D} .
$$

By [9, Lemma 2.3], we have that $f_{a} \in \mathscr{L}_{p, \lambda}$ and $\sup _{a \in \mathbb{D}}\left\|f_{a}\right\|_{\mathscr{L}_{p, \lambda}} \lesssim 1$. Fixed an arc $I \subset \partial \mathbb{D}$. Let $e^{i \theta}$ be the center of $I$ and $a=(1-|I|) e^{i \theta}$. Then

$$
|1-\bar{a} z| \asymp 1-|a|=|I|,\left|f_{a}(z)\right| \asymp|I|^{\frac{\lambda-1}{p}},
$$

whenever $z \in S(I)$. So

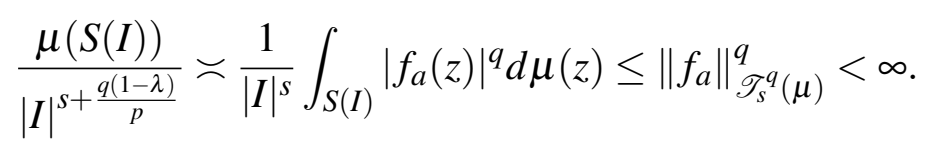

Therefore, $\mu$ is a $\left(s+\frac{q(1-\lambda)}{p}\right)$-Carleson measure. 
Conversely, suppose that $\mu$ is a $\left(s+\frac{q(1-\lambda)}{p}\right)$-Carleson measure. Fix $f \in \mathscr{L}_{p, \lambda}$. Let $I$ be any arc on $\partial \mathbb{D}$ and $a=(1-|I|) e^{i \theta}$, where $e^{i \theta}$ is the midpoint of $I$. From Lemma 2.2, we have

$$
|f(a)| \lesssim \frac{\|f\|_{\mathscr{L}_{p, \lambda}}}{(1-|a|)^{\frac{1-\lambda}{p}}}=\frac{\|f\|_{\mathscr{L}_{p, \lambda}}}{|I|^{\frac{1-\lambda}{p}}} .
$$

By using the triangle inequality, we deduce that

$$
\begin{aligned}
\frac{1}{|I|^{s}} \int_{S(I)}|f(z)|^{q} d \mu(z) & \lesssim \frac{1}{|I|^{s}} \int_{S(I)}|f(a)|^{q} d \mu(z)+\frac{1}{|I|^{s}} \int_{S(I)}|f(z)-f(a)|^{q} d \mu(z) \\
& =I_{1}+I_{2} .
\end{aligned}
$$

It is obvious that

$$
I_{1} \lesssim \frac{\mu(S(I))}{|I|^{s+\frac{q(1-\lambda)}{p}}}\|f\|_{\mathscr{L}_{p, \lambda}}^{q} \lesssim\|f\|_{\mathscr{L}_{p, \lambda}}^{q} .
$$

Since $s>\frac{q \lambda}{p}$, we obtain $s+\frac{q(1-\lambda)}{p}>1$. By the assumed condition and Theorem 7.4 in [12], we know that $i: A_{\frac{p s}{q}-1-\lambda}^{p} \rightarrow L^{q}(d \mu)$ is bounded. Note that

$$
\mathscr{L}_{p, \lambda} \subseteq H^{p} \subseteq A_{\frac{p s}{q}-1-\lambda}^{p}
$$

Based on these facts, we turn to estimate $I_{2}$. The estimate will be divided into two cases.

Case 1: $\frac{p s}{q}-\lambda \geq 1$.

$$
\begin{aligned}
& I_{2} \asymp \int_{S(I)} \frac{|f(z)-f(a)|^{q}}{|1-\bar{a} z|^{s}} d \mu(z) \\
& \asymp\left(1-|a|^{2}\right)^{\frac{(3-\lambda) q}{p}} \int_{S(I)} \frac{|f(z)-f(a)|^{q}}{|1-\bar{a} z|^{\frac{(3-\lambda) q}{p}+s}} d \mu(z) \\
& \lesssim\left(1-|a|^{2}\right)^{\frac{(3-\lambda) q}{p}} \int_{\mathbb{D}} \frac{|f(z)-f(a)|^{q}}{\left|(1-\bar{a} z)^{\frac{3-\lambda}{p}+\frac{s}{q}}\right|^{q}} d \mu(z) \\
& \lesssim\left(1-|a|^{2}\right)^{\frac{(3-\lambda) q}{p}-\frac{2 q}{p}}\left(\int_{\mathbb{D}} \frac{|f(z)-f(a)|^{p}\left(1-|a|^{2}\right)^{2}}{|1-\bar{a} z|^{3-\lambda+\frac{p s}{q}}}\left(1-|z|^{2}\right)^{\frac{p s}{q}-1-\lambda} d A(z)\right)^{q / p} \\
& \lesssim\left(1-|a|^{2}\right)^{\frac{(3-\lambda) q}{p}-\frac{2 q}{p}}\left(\int_{\mathbb{D}} \frac{|f(z)-f(a)|^{p}\left(1-|a|^{2}\right)^{2}}{|1-\bar{a} z|^{4}} d A(z)\right)^{q / p} \\
& =\left(\left(1-|a|^{2}\right)^{3-\lambda-2} \int_{\mathbb{D}} \frac{|f(z)-f(a)|^{p}\left(1-|a|^{2}\right)^{2}}{|1-\bar{a} z|^{4}} d A(z)\right)^{q / p} \\
& =\left(\left(1-|a|^{2}\right)^{1-\lambda} \int_{\mathbb{D}}\left|f \circ \varphi_{a}(w)-f(a)\right|^{p} d A(w)\right)^{q / p} \\
& \lesssim\left(\left(1-|a|^{2}\right)^{1-\lambda} \int_{\partial \mathbb{D}}\left|f \circ \varphi_{a}(\zeta)-f(a)\right|^{p} d \zeta\right)^{q / p} \\
& \leq\|f\|_{\mathscr{L}_{p, \lambda}}^{q}<\infty .
\end{aligned}
$$

The last second inequality is from [13, Theorem 1]. 
Case 2: $0<\frac{p s}{q}-\lambda<1$.

$$
\begin{aligned}
I_{2} & \asymp\left(1-|a|^{2}\right)^{-s} \int_{S(I)}|f(z)-f(a)|^{q} d \mu(z) \\
& \asymp\left(1-|a|^{2}\right)^{\frac{4 q}{p}-s} \int_{S(I)} \frac{|f(z)-f(a)|^{q}}{|1-\bar{a} z|^{\frac{4 q}{p}}} d \mu(z) \\
& \lesssim\left(1-|a|^{2}\right)^{\frac{4 q}{p}-s} \int_{\mathbb{D}} \frac{|f(z)-f(a)|^{q}}{|1-\bar{a} z|^{\frac{4 q}{p}}} d \mu(z) \\
& \lesssim\left(1-|a|^{2}\right)^{\frac{4 q}{p}-\frac{2 q}{p}-s}\left(\int_{\mathbb{D}} \frac{|f(z)-f(a)|^{p}\left(1-|a|^{2}\right)^{2}}{|1-\bar{a} z|^{4}}\left(1-|z|^{2}\right)^{\frac{p s}{q}-\lambda-1} d A(z)\right)^{q / p} \\
& =\left(\left(1-|a|^{2}\right)^{2-\frac{p s}{q}} \int_{\mathbb{D}}\left|f \circ \varphi_{a}(w)-f(a)\right|^{p}\left(1-\left|\varphi_{a}(w)\right|^{2}\right)^{\frac{p s}{q}-\lambda-1} d A(w)\right)^{q / p} \\
& \lesssim\left(\left(1-|a|^{2}\right)^{1-\lambda} \int_{\mathbb{D}}\left|f \circ \varphi_{a}(w)-f(a)\right|^{p}\left(1-|w|^{2}\right)^{\frac{p s}{q}-\lambda-1} d A(w)\right)^{q / p} \\
& \lesssim\left(\left(1-|a|^{2}\right)^{1-\lambda} \int_{\partial \mathbb{D}}\left|f \circ \varphi_{a}(\zeta)-f(a)\right|^{p} d \zeta\right)^{q / p} \\
& \lesssim\|f\|_{\mathscr{L}_{p, \lambda}}^{q}<\infty .
\end{aligned}
$$

Combining the estimates $I_{1}$ and $I_{2}$, we conclude that the identity mapping $i: \mathscr{L}_{p, \lambda} \rightarrow \mathscr{T}_{s}^{q}(\mu)$ is bounded.

Recall that the identity mapping $i: \mathscr{L}_{p, \lambda} \rightarrow \mathscr{T}_{s}^{q}(\mu)$ is compact if

$$
\lim _{n \rightarrow \infty} \frac{1}{|I|^{s}} \int_{S(I)}\left|f_{n}(z)\right|^{q} d \mu(z)=0
$$

whenever $I \subseteq \partial \mathbb{D}$ and $\left\{f_{n}\right\}$ is a bounded sequence in $\mathscr{L}_{p, \lambda}$ that converges to 0 uniformly on compact subsets of $\mathbb{D}$.

Theorem 2.2. Let $\mu$ be a positive Borel measure on $\mathbb{D}$. Let $1 \leq p<q<\infty, 0<\lambda<1$ and $\frac{q \lambda}{p}<s<\infty$. Then the identity mapping $i: \mathscr{L}_{p, \lambda} \rightarrow \mathscr{T}_{s}^{q}(\mu)$ is compact if and only if

$$
\lim _{|I| \rightarrow 0} \frac{\mu(S(I))}{|I|^{s+\frac{q(1-\lambda)}{p}}}=0 .
$$

Proof. Assume that $i: \mathscr{L}_{p, \lambda} \rightarrow \mathscr{T}_{s}^{q}(\mu)$ is compact. Give a sequence of $\operatorname{arcs}\left\{I_{n}\right\}$ with $\lim _{n \rightarrow \infty}\left|I_{n}\right|=$ 0 . Denote the center of $I_{n}$ by $e^{i \theta_{n}}$ and $a_{n}=\left(1-\left|I_{n}\right|\right) e^{i \theta_{n}}$. Set

$$
f_{n}(z)=\frac{\left(1-\left|a_{n}\right|^{2}\right)^{1+\frac{\lambda-1}{p}}}{\left(1-\overline{a_{n}} z\right)}, z \in \mathbb{D} .
$$

It is clear that $\left\{f_{n}\right\}$ is bounded in $\mathscr{L}_{p, \lambda}$ and $\left\{f_{n}\right\}$ converges to zero uniformly on any compact subset of $\mathbb{D}$. Then $\lim _{n \rightarrow \infty}\left\|f_{n}\right\|_{\mathscr{T}_{s}^{q}(\mu)}=0$. Since

$$
\left|f_{n}(z)\right| \asymp\left(1-\left|a_{n}\right|\right)^{\frac{\lambda-1}{p}}=\left|I_{n}\right|^{\frac{\lambda-1}{p}}, z \in S\left(I_{n}\right),
$$


we obtain

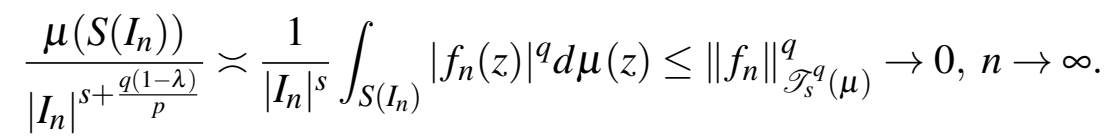

Then $\mu$ is a vanishing $\left(s+\frac{q(1-\lambda)}{p}\right)$-Carleson measure by the arbitrariness of $\left\{I_{n}\right\}$.

Conversely, suppose that $\mu$ is a vanishing $\left(s+\frac{q(1-\lambda)}{p}\right)$-Carleson measure. Then $\mu$ is also a $\left(s+\frac{q(1-\lambda)}{p}\right)$-Carleson measure and $\lim _{r \rightarrow 1^{-}}\left\|\mu-\mu_{r}\right\|_{s+\frac{q(1-\lambda)}{p}}=0$ by Lemma 2.3. It follows from the boundedness above, the mapping $i: \mathscr{L}_{p, \lambda} \rightarrow \mathscr{T}_{s}^{q}(\mu)$ is bounded. Let $\left\{f_{n}\right\}$ be a bounded sequence in $\mathscr{L}_{p, \lambda}$ such that $\left\{f_{n}\right\}$ converges to zero uniformly on each compact subset of $\mathbb{D}$. We have

$$
\begin{aligned}
\frac{1}{|I|^{s}} \int_{S(I)}\left|f_{n}(z)\right|^{q} d \mu(z) & \lesssim \frac{1}{|I|^{s}} \int_{S(I)}\left|f_{n}(z)\right|^{q} d \mu_{r}(z)+\frac{1}{|I|^{s}} \int_{S(I)}\left|f_{n}(z)\right|^{q} d\left(\mu-\mu_{r}\right)(z) \\
& \lesssim \frac{1}{|I|^{s}} \int_{S(I)}\left|f_{n}(z)\right|^{q} d \mu_{r}(z)+\left\|\mu-\mu_{r}\right\|_{S+\frac{q(1-\lambda)}{p}}\left\|f_{n}\right\|_{\mathscr{L}_{p, \lambda}}^{q} \\
& \lesssim \frac{1}{|I|^{s}} \int_{S(I)}\left|f_{n}(z)\right|^{q} d \mu_{r}(z)+\left\|\mu-\mu_{r}\right\|_{s+\frac{q(1-\lambda)}{p}} \\
& \rightarrow 0
\end{aligned}
$$

as $r \rightarrow 1^{-}$and $n \rightarrow \infty$. Therefore, $\lim _{n \rightarrow \infty}\left\|f_{n}\right\|_{\mathscr{T}_{s}^{q}(\mu)}=0$. This shows that $i: \mathscr{L}_{p, \lambda} \rightarrow \mathscr{T}_{s}^{q}(\mu)$ is compact.

\section{BOUNDEDNESS OF $T_{g}, I_{g}$ AND $M_{g}$}

Let $f, g \in \mathscr{H}(\mathbb{D})$. The Volterra integral operator $T_{g}$ and the integral operator $I_{g}$ are defined by

$$
T_{g} f(z)=\int_{0}^{z} g^{\prime}(w) f(w) d w, I_{g} f(z)=\int_{0}^{z} g(w) f^{\prime}(w) d w, z \in \mathbb{D}
$$

respectively.

There are many interesting results associated with $T_{g}$. For example, it was showed that $T_{g}$ is bounded on Hardy spaces if and only if $g \in B M O A$ (see, e.g., [14, 15]). In [16], Aleman and Siskakis showed that $T_{g}$ is bounded on the Bergman space $A^{p}$ if and only if $g \in \mathscr{B}$. In [17], Siskakis and Zhao proved that $T_{g}: B M O A \rightarrow B M O A$ is bounded if and only if $g \in B M O A_{\log }$. Pau and Peláez [18] and Xiao [10] studied $T_{g}$ acting on $Q_{p}$, respectively. For more information on operator $T_{g}$, see $[3,8,11,14,16,17,19,20,21,22,23,24]$ and the references therein.

In this section, via the embedding theorem (Theorem 2.2), we provide the characterizations for the boundedness of Volterra integral operator $T_{g}$ and its companion operator $I_{g}$ from $\mathscr{L}_{p, \lambda}$ to $F\left(q, q-2+\frac{q(1-\lambda)}{p}, s\right)$. Moreover, we study the multiplication operator $M_{g}$ from $\mathscr{L}_{p, \lambda}$ to $F\left(q, q-2+\frac{q(1-\lambda)}{p}, s\right)$.

Theorem 3.1. Let $g \in \mathscr{H}(\mathbb{D}), 1 \leq p<q<\infty, 0<\lambda<1$ and $\frac{q \lambda}{p}<s<\infty$. Then $T_{g}: \mathscr{L}_{p, \lambda} \rightarrow$ $F\left(q, q-2+\frac{q(1-\lambda)}{p}, s\right)$ is bounded if and only if $g \in \mathscr{B}$. Furthermore, $\left\|T_{g}\right\| \asymp\|g\|_{\mathscr{B}}$. 
Proof. Let $g \in \mathscr{B}$. Using the equivalent norm of Bloch function, we obtain

$$
\begin{aligned}
\|g\|_{\mathscr{B}}^{q} & \asymp \sup _{a \in \mathbb{D}} \int_{\mathbb{D}}\left|g^{\prime}(z)\right|^{q}\left(1-|z|^{2}\right)^{q-2}\left(1-\left|\varphi_{a}(z)\right|^{2}\right)^{s+\frac{q(1-\lambda)}{p}} d A(z) \\
& =\sup _{a \in \mathbb{D}} \int_{\mathbb{D}}\left|g^{\prime}(z)\right|^{q}\left(1-|z|^{2}\right)^{q-2+s+\frac{q(1-\lambda)}{p}}\left(\frac{1-|a|^{2}}{|1-\bar{a} z|^{2}}\right)^{s+\frac{q(1-\lambda)}{p}} d A(z) \\
& \asymp \sup _{I \subset \partial \mathbb{D}} \frac{1}{|I|^{s+\frac{q(1-\lambda)}{p}}} \int_{S(I)}\left|g^{\prime}(z)\right|^{q}\left(1-|z|^{2}\right)^{q-2+s+\frac{q(1-\lambda)}{p}} d A(z) .
\end{aligned}
$$

This means that

$$
d \mu_{g}(z)=\left|g^{\prime}(z)\right|^{p}\left(1-|z|^{2}\right)^{q-2+s+\frac{q(1-\lambda)}{p}} d A(z)
$$

is a $\left(s+\frac{q(1-\lambda)}{p}\right)$-Carleson measure. From Theorem 2.2, we find that $i: \mathscr{L}_{p, \lambda} \rightarrow \mathscr{T}_{s}^{q}\left(\mu_{g}\right)$ is bounded. Let $f \in \mathscr{L}_{p, \lambda}$. We deduce that

$$
\begin{aligned}
\left\|T_{g} f\right\|_{F\left(q, q-2+\frac{q(1-\lambda)}{p}, s\right)}^{q} & =\sup _{a \in \mathbb{D}} \int_{\mathbb{D}}|f(z)|^{q}\left|g^{\prime}(z)\right|^{q}\left(1-|z|^{2}\right)^{q-2+\frac{q(1-\lambda)}{p}}\left(1-\left|\varphi_{a}(z)\right|^{2}\right)^{s} d A(z) \\
& =\sup _{a \in \mathbb{D}} \int_{\mathbb{D}}|f(z)|^{q}\left|g^{\prime}(z)\right|^{q}\left(1-|z|^{2}\right)^{q-2+s+\frac{q(1-\lambda)}{p}}\left(\frac{1-|a|^{2}}{|1-\bar{a} z|^{2}}\right)^{s} d A(z) \\
& \asymp \sup _{I \subset \partial \mathbb{D}} \frac{1}{|I|^{s}} \int_{S(I)}|f(z)|^{q} d \mu_{g}(z) \\
& =\|f\|_{\mathscr{S}_{s}^{q}\left(\mu_{g}\right)}^{q} \\
& \lesssim\left\|\mu_{g}\right\|_{s+\frac{q(1-\lambda)}{p}}\|f\|_{\mathscr{L}_{p, \lambda}}^{q} \\
& \asymp\|g\|_{\mathscr{B}}^{q}\|f\|_{\mathscr{L}_{p, \lambda}}^{q} .
\end{aligned}
$$

That is, $T_{g}: \mathscr{L}_{p, \lambda} \rightarrow F\left(q, q-2+\frac{q(1-\lambda)}{p}, s\right)$ is bounded and $\left\|T_{g}\right\| \lesssim\|g\|_{\mathscr{B}}$.

Suppose that $T_{g}: \mathscr{L}_{p, \lambda} \rightarrow F\left(q, q-2+\frac{q(1-\lambda)}{p}, s\right)$ is bounded. Let $a \in \mathbb{D}$ and $f_{a}$ be as in (2.1). Then $f_{a} \in \mathscr{L}_{p, \lambda}$ and $\left\|f_{a}\right\|_{\mathscr{L}_{p, \lambda}} \lesssim 1$. It follows that

$$
\left\|T_{g} f_{a}\right\|_{F\left(q, q-2+\frac{q(1-\lambda)}{p}, s\right)} \leq\left\|T_{g}\right\|\left\|f_{a}\right\|_{\mathscr{L}_{p, \lambda}} \lesssim\left\|T_{g}\right\| .
$$

By Lemma 4.12 of [12], we have

$$
\begin{aligned}
& \|\left. T_{g} f_{a}\right|_{F\left(q, q-2+\frac{q(1-\lambda)}{p}, s\right)} ^{q} \\
\geq & \int_{\mathbb{D}}\left|g^{\prime}(z)\right|^{q} \frac{\left(1-|a|^{2}\right)^{q+\frac{q(\lambda-1)}{p}}}{|1-\bar{a} z|^{q}}\left(1-|z|^{2}\right)^{q-2+\frac{q(1-\lambda)}{p}}\left(1-\left|\varphi_{a}(z)\right|^{2}\right)^{s} d A(z) \\
\geq & \int_{\mathbb{D}(a, r)}\left|g^{\prime}(z)\right|^{q} \frac{\left(1-|a|^{2}\right)^{q+\frac{q(\lambda-1)}{p}}}{|1-\bar{a} z|^{q}}\left(1-|z|^{2}\right)^{q-2+\frac{q(1-\lambda)}{p}}\left(1-\left|\varphi_{a}(z)\right|^{2}\right)^{s} d A(z) \\
\gtrsim & \left|g^{\prime}(a)\right|^{q}\left(1-|a|^{2}\right)^{q} .
\end{aligned}
$$


Here $\mathbb{D}(a, r)=\{z \in \mathbb{D}: \beta(a, z)<r\}$ denotes the Bergman metric disk centered at $a$ with radius $r$ (see [12]). It follows that

$$
\left|g^{\prime}(a)\right|\left(1-|a|^{2}\right) \lesssim\left\|T_{g} f_{a}\right\|_{F\left(q, q-2+\frac{q(1-\lambda)}{p}, s\right)} \lesssim\left\|T_{g}\right\| .
$$

Thus, $g \in \mathscr{B}$ and $\|g\|_{\mathscr{B}} \lesssim\left\|T_{g}\right\|$.

Theorem 3.2. Suppose that $2 \leq p<q<\infty, 0<\lambda<1$ and $\lambda<s<2$. If $g \in \mathscr{H}(\mathbb{D})$, then $I_{g}$ : $\mathscr{L}_{p, \lambda} \rightarrow F\left(q, q-2+\frac{q(1-\lambda)}{p}, s\right)$ is bounded if and only if $g \in \mathscr{H}^{\infty}$. Furthermore, $\left\|I_{g}\right\| \asymp\|g\|_{\mathscr{H}^{\infty}}$.

Proof. Suppose that $g \in \mathscr{H}^{\infty}$ and $f \in \mathscr{L}_{p, \lambda}$. For any $a \in \mathbb{D}$, we have

$$
\left|f^{\prime}(z)\right|^{q} \lesssim \frac{\|f\|_{\mathscr{L}_{p, \lambda}}^{q}}{\left(1-|z|^{2}\right)^{q+\frac{q(1-\lambda)}{p}}} .
$$

From [25, Theorem 1], we have

$$
\|\cdot\|_{F(p, p-1-\lambda, \lambda)} \lesssim\|\cdot\|_{\mathscr{L}_{p, \lambda}} .
$$

Thus,

$$
\begin{aligned}
\left\|I_{g} f\right\|_{F\left(q, q-2+\frac{q(1-\lambda)}{p}, s\right)}^{q} & \leq\|g\|_{\mathscr{H}^{\infty}}^{q} \sup _{a \in \mathbb{D}} \int_{\mathbb{D}}\left|f^{\prime}(z)\right|^{q}\left(1-|z|^{2}\right)^{q-2+\frac{q(1-\lambda)}{p}}\left(1-\left|\varphi_{a}(z)\right|^{2}\right)^{s} d A(z) \\
& \leq\|g\|_{\mathscr{H}^{\infty}}^{q} \sup _{a \in \mathbb{D}} \int_{\mathbb{D}}\left|f^{\prime}(z)\right|^{p}\left(1-|z|^{2}\right)^{p-2+1-\lambda}\left(1-\left|\varphi_{a}(z)\right|^{2}\right)^{\lambda} d A(z) \\
& \leq\|g\|_{\mathscr{H}^{\infty}}^{q} \sup _{a \in \mathbb{D}} \int_{\mathbb{D}}\left|f^{\prime}(z)\right|^{p}\left(1-|z|^{2}\right)^{p-1-\lambda}\left(1-\left|\varphi_{a}(z)\right|^{2}\right)^{\lambda} d A(z) \\
& \lesssim\|g\|_{\mathscr{H}^{\infty}}^{q}\|f\|_{\mathscr{L}_{p, \lambda}}^{p} .
\end{aligned}
$$

Conversely, Let $a \in \mathbb{D}$ and

$$
F_{a}=\frac{\left(1-|a|^{2}\right)^{1+\frac{\lambda-1}{p}}}{\bar{a}(1-\bar{a} z)} \in \mathscr{L}_{p, \lambda} .
$$

It is easily to see that $\sup _{a \in \mathbb{D}}\left\|F_{a}\right\|_{\mathscr{L}_{p, \lambda}} \lesssim 1$ and hence

$$
\left\|I_{g} F_{a}\right\|_{F\left(q, q-2+\frac{q(1-\lambda)}{p}, s\right)} \leq\left\|I_{g}\right\|\left\|F_{a}\right\|_{\mathscr{L}_{p, \lambda}} \lesssim\left\|I_{g}\right\| .
$$

Furthermore, Lemma 4.12 of [12] gives

$$
\begin{aligned}
& \left\|I_{g} F_{a}\right\|_{F\left(q, q-2+\frac{q(1-\lambda)}{p}, s\right)}^{q} \\
\gtrsim & \int_{\mathbb{D}}|g(z)|^{q} \frac{\left(1-|a|^{2}\right)^{q+\frac{q(\lambda-1)}{p}}}{|1-\bar{a} z|^{2 q}}\left(1-|z|^{2}\right)^{q-2+\frac{q(1-\lambda)}{p}}\left(1-\left|\varphi_{a}(z)\right|^{2}\right)^{s} d A(z) \\
\gtrsim & \int_{\mathbb{D}(a, r)}|g(z)|^{q} \frac{\left(1-|a|^{2}\right)^{q+\frac{q(\lambda-1)}{p}}}{|1-\bar{a} z|^{2 q}}\left(1-|z|^{2}\right)^{q-2+\frac{q(1-\lambda)}{p}}\left(1-\left|\varphi_{a}(z)\right|^{2}\right)^{s} d A(z) \\
\gtrsim & |g(a)|^{q} .
\end{aligned}
$$

Therefore, $|g(a)| \lesssim\left\|I_{g}\right\|$. By the choice of $a$, we deduce that $g \in \mathscr{H}^{\infty}$ and $\|g\|_{\mathscr{H}^{\infty}} \lesssim\left\|I_{g}\right\|$. 
Recall that $\left(X,\|\cdot\|_{X}\right)$ and $\left(Y,\|\cdot\|_{Y}\right)$ are analytic function spaces. Denote by $M(X, Y)$ the set of multipliers from $X$ to $Y$, that is,

$$
M(X, Y)=\{g \in \mathscr{H}(\mathbb{D}): f g \in Y, \forall f \in X\} .
$$

The multiplication operator $M_{g}$ is defined by

$$
M_{g} f(z)=g(z) f(z), f \in \mathscr{H}(\mathbb{D}), z \in \mathbb{D} .
$$

The operators $T_{g}$ and $I_{g}$ are closely related to $M_{g}$ due to

$$
T_{g} f+I_{g} f=M_{g} f-f(0) g(0) .
$$

Using Theorems 3.1 and 3.2, we characterize the multipliers from $\mathscr{L}_{p, \lambda}$ to $F(q, q-2+$ $\left.\frac{q(1-\lambda)}{p}, s\right)$.

Theorem 3.3. Suppose that $2 \leq p<q<\infty, 0<\lambda<1$ and $\frac{q \lambda}{p}<s<2$. Then $M\left(\mathscr{L}_{p, \lambda}, F(q, q-\right.$ $\left.\left.2+\frac{q(1-\lambda)}{p}, s\right)\right)=\mathscr{H}^{\infty}$.

Proof. Let $g \in \mathscr{H}^{\infty}$. It follows from Theorems 3.1 and 3.2 that

$$
T_{g}: \mathscr{L}_{p, \lambda} \rightarrow F\left(q, q-2+\frac{q(1-\lambda)}{p}, s\right) \text { and } I_{g}: \mathscr{L}_{p, \lambda} \rightarrow F\left(q, q-2+\frac{q(1-\lambda)}{p}, s\right)
$$

are bounded. So $M_{g}: \mathscr{L}_{p, \lambda} \rightarrow F\left(q, q-2+\frac{q(1-\lambda)}{p}, s\right)$ is bounded.

Conversely, let $f \in F\left(q, q-2+\frac{q(1-\lambda)}{p}, s\right)$ and $a \in \mathbb{D}$. It follows that

$$
\left|f^{\prime}(a)\right| \lesssim \frac{\|f\|_{F\left(q, q-2+\frac{q(1-\lambda)}{p}, s\right)}}{\left(1-|a|^{2}\right)^{1+\frac{1-\lambda}{p}}}
$$

Since $a$ is arbitrary, we get

$$
|f(a)| \lesssim \frac{\|f\|_{F\left(q, q-2+\frac{q(1-\lambda)}{p}, s\right)}}{\left(1-|a|^{2}\right)^{\frac{1-\lambda}{p}}}
$$

For any $a \in \mathbb{D}$, let $f_{a}$ be defined as in (2.1). Then $\left\{f_{a}\right\}$ is bounded in $\mathscr{L}_{p, \lambda}$. It follows that $M_{g} f_{a} \in F\left(q, q-2+\frac{q(1-\lambda)}{p}, s\right)$ and then

$$
\left|M_{g} f_{a}(z)\right| \lesssim \frac{\left\|M_{g} f_{a}\right\|_{F\left(q, q-2+\frac{q(1-\lambda)}{p}, s\right)}}{\left(1-|z|^{2}\right)^{\frac{1-\lambda}{p}}} \lesssim \frac{\left\|M_{g}\right\|\left\|f_{a}\right\|_{\mathscr{L}_{p, \lambda}}}{\left(1-|z|^{2}\right)^{\frac{1-\lambda}{p}}} \lesssim \frac{\left\|M_{g}\right\|}{\left(1-|z|^{2}\right)^{\frac{1-\lambda}{p}}} .
$$

As a consequence,

$$
\left|\frac{1-|a|^{2}}{(1-\bar{a} z)^{1+\frac{1-\lambda}{p}}} g(z)\right| \lesssim \frac{\left\|M_{g}\right\|}{\left(1-|z|^{2}\right)^{\frac{1-\lambda}{p}}} .
$$

Using the arbitrariness of $z, a \in \mathbb{D}$, and letting $a=z$, we conclude that $g \in \mathscr{H}^{\infty}$ and $\|g\|_{\mathscr{H}^{\infty}} \lesssim$ $\left\|M_{g}\right\|$. 


\section{ESSENTIAL NORM OF $T_{g}$ AND $I_{g}$}

Firstly, let us recall some definitions. Let $\left(X,\|\cdot\|_{X}\right)$ and $\left(Y,\|\cdot\|_{Y}\right)$ be Banach spaces and $T: X \rightarrow Y$ be a bounded linear operator. The essential norm of $T: X \rightarrow Y$, denoted by $\|T\|_{e}$, is defined by

$$
\|T\|_{e}=\inf _{K}\left\{\|T-K\|_{X \rightarrow Y}: K \text { is compact from } X \text { to } Y\right\} .
$$

It is clear that $T: X \rightarrow Y$ is compact if and only if $\|T\|_{e}=0$. Let $\Omega$ be a closed subspace of $X$. Given $f \in X$, the distance from $f$ to $\Omega$, denoted by $\operatorname{dist}_{X}(f, \Omega)$, is defined by

$$
\operatorname{dist}_{X}(f, \Omega)=\inf _{g \in \Omega}\|f-g\|_{X} .
$$

Lemma 4.1. $[26,27]$ If $f \in \mathscr{B}$, then

$$
\limsup _{|z| \rightarrow 1^{-}}\left(1-|z|^{2}\right)\left|f^{\prime}(z)\right| \asymp \operatorname{dist}_{\mathscr{B}}\left(f, \mathscr{B}_{0}\right) \asymp \limsup _{r \rightarrow 1^{-}}\left\|f-f_{r}\right\|_{\mathscr{B}},
$$

where $f_{r}(z)=f(r z), 0<r<1, z \in \mathbb{D}$.

We need the following lemma.

Lemma 4.2. Let $1 \leq p<q<\infty, 0<\lambda<1$ and $\frac{q \lambda}{p}<s<\infty$. If $0<r<1$ and $g \in \mathscr{B}$, then $T_{g_{r}}: \mathscr{L}_{p, \lambda} \rightarrow F\left(q, q-2+\frac{q(1-\lambda)}{p}, s\right)$ is compact.

Proof. Given $\left\{f_{n}\right\} \subset \mathscr{L}_{p, \lambda}$ such that $\left\{f_{n}\right\}$ converges to zero uniformly on any compact subset of $\mathbb{D}$ and $\sup _{n}\left\|f_{n}\right\|_{\mathscr{L}_{p, \lambda}} \leq 1$, for each $a \in \mathbb{D}$, we have

$$
\begin{aligned}
& \left\|T_{g_{r}} f_{n}\right\|_{F\left(q, q-2+\frac{q(1-\lambda)}{p}, s\right)}^{q} \\
& =\sup _{a \in \mathbb{D}} \int_{\mathbb{D}}\left|f_{n}(z)\right|^{q}\left|g_{r}^{\prime}(z)\right|^{q}\left(1-|z|^{2}\right)^{q-2+\frac{q(1-\lambda)}{p}}\left(1-\left|\varphi_{a}(z)\right|^{2}\right)^{s} d A(z) \\
& \lesssim \frac{\|g\|_{\mathscr{B}}^{q}}{\left(1-r^{2}\right)^{q}} \sup _{a \in \mathbb{D}} \int_{\mathbb{D}}\left|f_{n}(z)\right|^{q}\left(1-|z|^{2}\right)^{q-2+\frac{q(1-\lambda)}{p}}\left(1-\left|\varphi_{a}(z)\right|^{2}\right)^{s} d A(z) \\
& \lesssim \frac{\|g\|_{\mathscr{B}}^{q}\left\|f_{n}\right\|_{\mathscr{L}_{p, \lambda}}^{q}}{\left(1-r^{2}\right)^{q}} \int_{\mathbb{D}}\left(1-|z|^{2}\right)^{q-2}\left(1-\left|\varphi_{a}(z)\right|^{2}\right)^{s} d A(z) \\
& \lesssim \frac{\|g\|_{\mathscr{B}}^{q}\left\|f_{n}\right\|_{\mathscr{L}_{p, \lambda}}^{q}}{\left(1-r^{2}\right)^{q}} .
\end{aligned}
$$

By the dominated convergence theorem, we get the desire result. The proof is complete.

Theorem 4.1. Let $1 \leq p<q<\infty, 0<\lambda<1$ and $\frac{q \lambda}{p}<s<\infty$. If $g \in \mathscr{H}(\mathbb{D})$ and $T_{g}: \mathscr{L}_{p, \lambda} \rightarrow$ $F\left(q, q-2+\frac{q(1-\lambda)}{p}, s\right)$ is bounded, then

$$
\left\|T_{g}\right\|_{e} \asymp \limsup _{|z| \rightarrow 1^{-}}\left(1-|z|^{2}\right)\left|g^{\prime}(z)\right| \asymp \operatorname{dist}_{\mathscr{B}}\left(g, \mathscr{B}_{0}\right) .
$$

Proof. Let $\left\{a_{n}\right\}$ be a sequence in $\mathbb{D}$ such that $\lim _{n \rightarrow \infty}\left|a_{n}\right|=1$. For each $n$, let $f_{n}$ be defined as in (2.2). Then $\left\{f_{n}\right\}$ is bounded in $\mathscr{L}_{p, \lambda}$. Furthermore, $\left\{f_{n}\right\}$ converges to zero uniformly on every 
compact subset of $\mathbb{D}$. Given a compact operator $K: \mathscr{L}_{p, \lambda} \rightarrow F\left(q, q-2+\frac{q(1-\lambda)}{p}, s\right)$, we have from [28] that $\lim _{n \rightarrow \infty}\left\|K f_{n}\right\|_{F\left(q, q-2+\frac{q(1-\lambda)}{p}, s\right)}=0$. So

$$
\begin{aligned}
\left\|T_{g}-K\right\| & \gtrsim \limsup _{n \rightarrow \infty}\left\|\left(T_{g}-K\right) f_{n}\right\|_{F\left(q, q-2+\frac{q(1-\lambda)}{p}, s\right)} \\
& \gtrsim \limsup _{n \rightarrow \infty}\left(\left\|T_{g} f_{n}\right\|_{F\left(q, q-2+\frac{q(1-\lambda)}{p}, s\right)}-\left\|K f_{n}\right\|_{F\left(q, q-2+\frac{q(1-\lambda)}{p}, s\right)}\right) \\
& =\limsup _{n \rightarrow \infty}\left\|T_{g} f_{n}\right\|_{F\left(q, q-2+\frac{q(1-\lambda)}{p}, s\right)} \\
& \geq \limsup _{n \rightarrow \infty}\left(\int_{\mathbb{D}}\left|f_{n}(z)\right|^{q}\left|g^{\prime}(z)\right|^{q}\left(1-|z|^{2}\right)^{q-2+\frac{q(1-\lambda)}{p}}\left(1-\left|\varphi_{a_{n}}(z)\right|^{2}\right)^{s} d A(z)\right)^{\frac{1}{q}} \\
& \gtrsim \limsup _{n \rightarrow \infty}\left(1-\left|a_{n}\right|^{2}\right)\left|g^{\prime}\left(a_{n}\right)\right|,
\end{aligned}
$$

which implies

$$
\left\|T_{g}\right\|_{e} \gtrsim \limsup _{n \rightarrow \infty}\left(1-\left|a_{n}\right|^{2}\right)\left|g^{\prime}\left(a_{n}\right)\right| \text {. }
$$

It follows from Lemma 4.1 and the arbitrariness of $\left\{a_{n}\right\}$ that

$$
\left\|T_{g}\right\|_{e} \gtrsim \limsup _{|z| \rightarrow 1^{-}}\left(1-|z|^{2}\right)\left|g^{\prime}(z)\right| \asymp \operatorname{dist}_{\mathscr{B}}\left(g, \mathscr{B}_{0}\right) .
$$

On the other hand, by Lemma 4.2, we have that $T_{g_{r}}: \mathscr{L}_{p, \lambda} \rightarrow F\left(q, q-2+\frac{q(1-\lambda)}{p}, s\right)$ is compact. Then

$$
\left\|T_{g}\right\|_{e} \leq\left\|T_{g}-T_{g_{r}}\right\|=\left\|T_{g-g_{r}}\right\| \asymp\left\|g-g_{r}\right\|_{\mathscr{B}} .
$$

Using Lemma 4.1 again, we have

$$
\left\|T_{g}\right\|_{e} \lesssim \limsup _{r \rightarrow 1^{-}}\left\|g-g_{r}\right\|_{\mathscr{B}} \asymp \operatorname{dist}_{\mathscr{B}}\left(g, \mathscr{B}_{0}\right)
$$

The proof is complete.

Using Theorem 4.1, we easily deduce the following corollary.

Corollary 4.1. Let $1 \leq p<q<\infty, 0<\lambda<1$ and $\frac{q \lambda}{p}<s<\infty$. If $g \in \mathscr{H}(\mathbb{D})$, then $T_{g}: \mathscr{L}_{p, \lambda} \rightarrow$ $F\left(q, q-2+\frac{q(1-\lambda)}{p}, s\right)$ is compact if and only if $g \in \mathscr{B}_{0}$.

Theorem 4.2. Suppose that $2 \leq p<q<\infty, 0<\lambda<1$ and $\lambda<s<2$. If $g \in \mathscr{H}(\mathbb{D})$ and $I_{g}: \mathscr{L}_{p, \lambda} \rightarrow F\left(q, q-2+\frac{q(1-\lambda)}{p}, s\right)$ is bounded, then $\left\|I_{g}\right\|_{e} \asymp\|g\|_{\mathscr{H}^{\infty}}$.

Proof. Let $\left\{a_{n}\right\},\left\{f_{n}\right\}$ and $K$ be defined as in the proof of Theorem 4.1. Since $K: \mathscr{L}_{p, \lambda} \rightarrow$ $F\left(q, q-2+\frac{q(1-\lambda)}{p}, s\right)$ is compact, we get

$$
\lim _{n \rightarrow \infty}\left\|K f_{n}\right\|_{F\left(q, q-2+\frac{q(1-\lambda)}{p}, s\right)}=0 .
$$


Hence,

$$
\begin{aligned}
\left\|I_{g}-K\right\| & \gtrsim \limsup _{n \rightarrow \infty}\left\|\left(I_{g}-K\right) f_{n}\right\|_{F\left(q, q-2+\frac{q(1-\lambda)}{p}, s\right)} \\
& \gtrsim \limsup _{n \rightarrow \infty}\left(\left\|I_{g} f_{n}\right\|_{F\left(q, q-2+\frac{q(1-\lambda)}{p}, s\right)}-\left\|K f_{n}\right\|_{F\left(q, q-2+\frac{q(1-\lambda)}{p}, s\right)}\right) \\
& =\limsup _{n \rightarrow \infty}\left\|I_{g} f_{n}\right\|_{F\left(q, q-2+\frac{q(1-\lambda)}{p}, s\right)} .
\end{aligned}
$$

Therefore,

$$
\left\|I_{g}\right\|_{e} \gtrsim \limsup _{n \rightarrow \infty}\left\|I_{g} f_{n}\right\|_{F\left(q, q-2+\frac{q(1-\lambda)}{p}, s\right)} .
$$

Similar argument as in the proof of Theorem 3.2 shows that

$$
\left\|I_{g} f_{n}\right\|_{F\left(q, q-2+\frac{q(1-\lambda)}{p}, s\right)}^{q} \gtrsim\left|g\left(a_{n}\right)\right|^{q},
$$

which implies that $\left\|I_{g}\right\|_{e} \gtrsim\|g\|_{\mathscr{H}^{\infty}}$.

On the other hand, Theorem 3.2 gives

$$
\left\|I_{g}\right\|_{e}=\inf _{K}\left\|I_{g}-K\right\| \leq\left\|I_{g}\right\| \lesssim\|g\|_{\mathscr{H}^{\infty}} .
$$

The proof is complete.

Corollary 4.2. Suppose that $2 \leq p<q<\infty, 0<\lambda<1$ and $\lambda<s<2$. If $g \in \mathscr{H}(\mathbb{D})$, then $I_{g}: \mathscr{L}_{p, \lambda} \rightarrow F\left(q, q-2+\frac{q(1-\lambda)}{p}, s\right)$ is compact if and only if $g=0$.

\section{Acknowledgments}

This work was supported by the NNSF of China (No. 11701222, 11801250, 11871257), the Overseas Scholarship Program for Elite Young and Middle-aged Teachers of Lingnan Normal University, the Key Program of Lingnan Normal University (No. LZ1905), China Postdoctoral Science Foundation (no. 2018M633090), the Innovation and developing School Project of Guangdong Province (No. 2019KZDXM032) and the Department of Education of Guangdong Province (No. 2018KTSCX133).

\section{REFERENCES}

[1] P. Duren, Theory of $H^{p}$ Spaces, Academic Press, New York, 1970.

[2] Z. Wu, C. Xie, $Q_{p}$ spaces and Morrey spaces, J. Funct. Anal. 201 (2003), 282-297.

[3] P. Li, J. Liu, Z. Lou, Integral operators on analytic Morrey spaces, Sci. China Math. 57 (2014), 1961-1974.

[4] J. Liu, Z. Lou, Carleson measure for analytic Morrey spaces, Nonlinear Anal. 125 (2015), 423-432.

[5] J. Garnett, Bounded Analytic Functions, Academic Press, New York, 1981.

[6] R. Zhao, On a general family of function spaces, Ann. Acad. Sci. Fenn. Math. Diss. No. 105 (1996), 1-56.

[7] J. Xiao, Holomorphic $Q$ Classes, Springer, LNM 1767, Berlin, 2001.

[8] J. Pau and R. Zhao, Carleson measures, Riemann-Stieltjes and multiplication operators on a general family of function spaces, Integr. Equ. Oper. Theory 78 (2014), 483-514.

[9] J. Wang, The Carleson measure problem between analytic Morrey spaces, Canad. Math. Bull. 59 (2016), 878-890.

[10] J. Xiao, The $Q_{p}$ Carleson measure problem, Adv. Math. 217 (2008), 2075-2088.

[11] S. Li, J. Liu, C. Yuan, Embedding theorems for Dirichlet type spaces, Canad. Math. Bull. 1 (2020), $106-117$.

[12] K. Zhu, Operator Theory in Function Spaces, Second Edition, Math. Surveys and Monographs 138, 2007.

[13] J. Xiao, C. Yuan, Analytic Campanato spaces and their compositions, Indian. Univ. Math. J. 64 (2015), 10011025 . 
[14] A. Aleman, A. Siskakis, An integral operator on $H^{p}$, Complex Var. Theory Appl. 28 (1995), 149-158.

[15] C. Pommerenke, Schlichte funktionen und analytische funktionen von beschränkten mittlerer Oszillation, Comm. Math. Helv. 52 (1977), 591-602.

[16] A. Aleman and A. Siskakis, Integration operators on Bergman spaces, Indiana Univ. Math. J. 46 (1997), 337-356.

[17] A. Siskakis, R. Zhao, A Volterra type operator on spaces of analytic functions, Contemp. Math. 232 (1999), 299-311.

[18] J. Pau, J. A. Peláez, Multipliers of Möbius invariant $Q_{s}$ spaces, Math. Z. 261 (2009), 545-555.

[19] A. Aleman, J. Cima, An integral operator on $H^{p}$ and Hardy's inequality, J. Anal. Math. 85 (2001), 157-176.

[20] S. Li, S. Stević, Riemann-Stieltjes operators between $\alpha$-Bloch spaces and Besov spaces, Math. Nachr. 282 (2009), 899-911.

[21] S. Li, S. Stević, Volterra type operators on Zygmund spaces, J. Inequal. Appl. 2007, Article ID 32124, 10 pp.

[22] S. Li, H. Wulan, Volterra type operators on $Q_{K}$ spaces, Taiwanese J. Math. 14 (2010), 195-211.

[23] R. Qian, S. Li, Volterra type operators on Morrey type spaces, Math. Inequal. Appl. 18 (2015), 1589-1599.

[24] Y. Shi, S. Li, Essential norm of integral operators on Morrey type spaces, Math. Inequal. Appl. 19 (2016), 385-393.

[25] C. Yuan, C. Tong, On Analytic Campanato and $F(p, q, s)$ Spaces, Complex Anal. Oper. Theory. 12 (2018), 1845-1875.

[26] K. Attele, Interpolating sequences for the derivatives of Bloch functions, Glasgow Math. J. 34 (1992), 35-41.

[27] M. Tjani, Distance of a Bloch function to the little Bloch space, Bull. Austral. Math. Soc. 74 (2006), 101-119.

[28] M. Tjani, Compact Composition Operators on Some Möbius Invariant Banach Spaces, PhD dissertation, Michigan State University, 1996. 